\title{
An Algorithm to Guide Recipient Vessel Selection in Cases of Free Functional Muscle Transfer for Facial Reanimation
}

\author{
Francis P Henry, Jonathan I Leckenby, Daniel P Butler, Adriaan O Grobbelaar \\ Department of Plastic and Reconstructive Surgery, The Royal Free Hospital, London, UK
}

Background The aim of this study was to review the recipient vessels used in our cases of facial reanimation with free functional muscle transfer and to identify patient variables that may predict when the facial vessels are absent. From this we present a protocol for vessel selection in cases when the facial artery and/or vein are absent.

Methods Patients were identified from November 2006 to October 2013. Data was collected on patient demographics, facial palsy aetiology, history of previous facial surgery/trauma and flap/recipient vessels used. A standard operative approach was adopted and performed by a single surgeon.

Results Eighty-seven eligible patients were identified for inclusion amongst which 98 hemifaces were operated upon. The facial artery and vein were the most commonly used recipient vessels ( $90 \%$ and $83 \%$ of patients, respectively). Commonly used alternative vessels were the transverse facial vein and superficial temporal artery. Those with congenital facial palsy were significantly more likely to lack a suitable facial vein $(P=0.03)$ and those with a history of previous facial surgery or trauma were significantly more likely to have an absent facial artery and vein $(P<0.05)$.

Conclusions Our algorithm can help to guide vessel selection cases of facial reanimation with free functional muscle transfer. Amongst patients with congenital facial palsy or in those with a previous history of facial surgery or trauma, the facial vessels are more likely to be absent and so the surgeon should then look towards the transverse facial vein and superficial temporal artery as alternative recipient structures.

Keywords Facial paralysis / Free tissue flaps / Microsurgery
Correspondence: Daniel Butler Department of Plastic and Reconstructive Surgery, The Royal Free Hospital, Pond Street, London NW3 20G, UK

Tel: +44-77-5947-3296

Fax: +44-20-7830-2195

E-mail: dan.butler@doctors.org.uk

This article was presented at the European Society of Plastic,

Reconstructive and Aesthetic Surgeons Meeting on July 7, 2014 in Edinburgh, UK.

No potential conflict of interest relevant to this article was reported.

\section{INTRODUCTION}

Facial palsy results in loss of movement on the affected side of the face with resulting aesthetic and functional complications. The resulting loss of facial expression can have a profound social impact. Free functional muscle transfer can be used to reanimate the affected hemiface and improve both static position and dynamic facial movements [1]. The benefits of free neurotised muscle transfer over regional muscle transfer techniques include the opportunity for the surgeon to have greater control over the positioning, tension and the direction of pull of the muscular tissue and, therefore, the appearance of the resulting smile. 
The concept of transferring distant muscle to the affected face was first developed by Thompson [2] in 1971. It wasn't until 1976 that Harii et al. [3] performed the first microneurovascular muscle transfer for facial reanimation using gracilis. The current practice in our unit is to use the pectoralis minor muscle due to its minimal bulk, good excursion and good functional outcomes [4].

For free tissue transfer to be successful suitable recipient vessels must be available. The position, calibre and handling characteristics are important variables when selecting an appropriate recipient artery and vein. Furthermore, vessel selection depends upon the length of the donor muscle vascular pedicle, which can be affected by the muscle chosen and the length of pedicle dissected free. Finally, suitable recipient vessels can be influenced by the pathology causing the facial palsy with previous facial trauma or surgery often distorting the facial vascular anatomy.

The arterial supply to the face consists of three main arterial trunks: facial; transverse facial and infraorbital. Our practice is to use the facial artery and vein as the recipient vessels. This is due to their direction of travel across the lower third of the face [5] and, therefore, proximity to the donor muscle vascular pedicle after inset which, in the case of a pectoralis minor muscle flap, can be very short [6].

The aim of this study was to review the recipient vessels used in our cases of facial reanimation with free functional muscle transfer and to identify patient variables that may predict the facial vessels are absent. From this we present a protocol for vessel selection in cases when the facial artery and/or vein are absent.

\section{Fig. 1. Superficial temporal and facial vessels}

An intraoperative image of a right hemiface during a second-stage facial reanimation procedure showing both the facial and superficial temporal vessels along with the distal ends of two cross-facial nerve grafts.

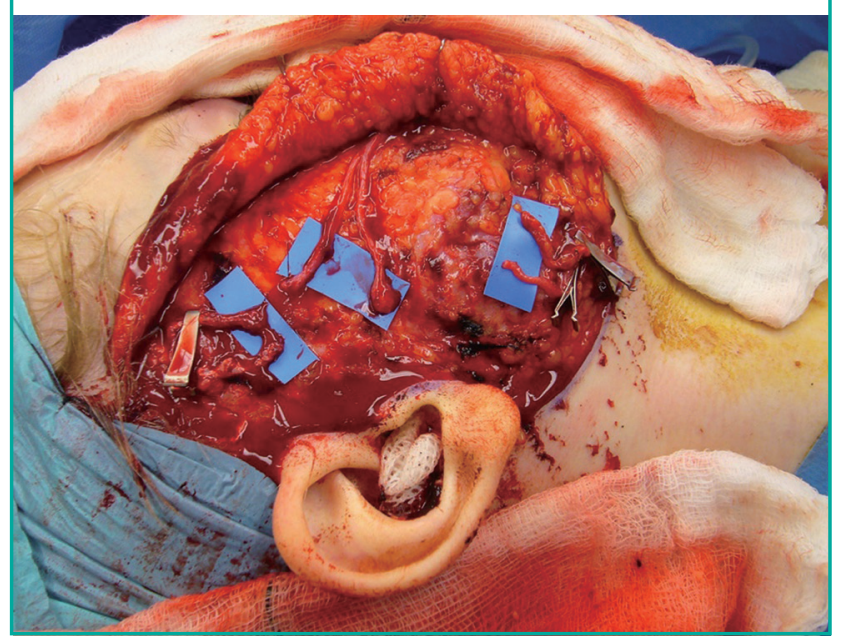

\section{METHODS}

All patients that underwent free functional muscle transfer for facial reanimation in the treatment of facial palsy between November 2006 and October 2013 were identified retrospectively using the departmental electronic patient system. Patients were excluded if their case notes were unavailable or documentation of the intraoperative findings was incomplete.

Data was collected on patient demographics, facial palsy aetiology, history of previous facial surgery/trauma and flap/recipient vessels used. All operations were performed by a single surgeon (A.O.G). When using a pectoralis minor or gracilis muscle flap a two-stage approach was used to reanimate the face, starting with a cross-facial nerve graft using the sural nerve as the donor nerve of choice. When using the latissimus dorsi muscle flap, a one-stage approach would often be used with the thoroacodorsal nerve coapted to the ipsilateral masseteric branch of the trigeminal nerve or a branch of the contralateral buccal branch of the facial nerve. Standard intraoperative procedure would involve an extended parotidectomy incision on the paralysed side of the face, elevation of the skin flap and exploration for the facial vessels. Exploration would start at the point where they cross the mandible just anterior to the masseter muscle given their consistent position at this point along their course [5]. In cases when a suitable facial artery and/or vein were not identified after detailed exploration, attempts would be made to identify alternative recipient vessels (Figs. 1, 2).

Analysis of data was performed using SPSS software and statistical comparison performed using the chi-square test. A P-val-

\section{Fig. 2. Transverse facial vein}

An intraoperative image of a right hemiface during a second-stage facial reanimation procedure showing the transverse facial vein resting on the green microsurgical background.

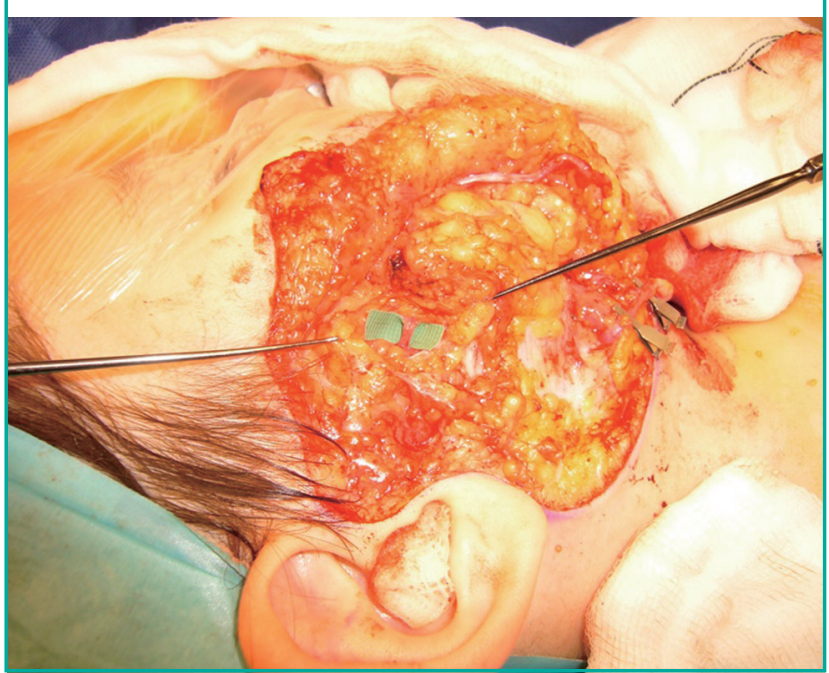


ue $<0.05$ was considered to be statistically significant.

\section{RESULTS}

A total of 103 patients had undergone facial reanimation during the study period. Eighty-seven were eligible for inclusion amongst which 98 hemifaces were operated upon. Of the eligible patients included in the study, $61 \%$ had congenital facial palsy (Table 1). Syndromic cases included those with Moebius syndrome $(n=13)$, CHARGE syndrome $(n=3)$, hemifacial microsomia $(n=2)$, Goldenhar syndrome $(n=1)$, first arch syndrome $(n=1)$ and Poland's syndrome $(n=1)$. Table 2 shows the distribution of free functional muscle transfers used. In a patient who had facial palsy secondary to previous debulking of extensive facial lymphatic malformations, the procedure had to be abandoned due to lack of any suitable recipient vessels. This particular patient had undergone resection of the internal and external jugular veins as part of the surgical resection and had undergone postoperative radiotherapy that made safe mobilisation of the external carotid artery for end-to-side anastomosis impossible. The recipient vessels used are shown in Tables 3 and 4.

After exclusion of patients that had undergone previous facial surgery or had suffered previous facial trauma $(n=18)$, it was found that those with congenital facial palsy were significantly more likely to have an absent facial vein on one or both sides of the face than those with acquired facial palsy ( $16 \%$ vs. $0 \%$ absent, $\mathrm{P}=0.03$ ). Amongst this cohort there was no difference in the proportion of patients with congenital or acquired facial palsy that had an absent facial artery on one or both sides of the face

Table 1. Facial palsy aetiology

\begin{tabular}{|lcc|}
\hline Aetiology group & Aetiology subgroup & No. of patients \\
\hline Congenital & - & 53 \\
& Nonsyndromic & 32 \\
Acquired & Syndromic & 21 \\
& - & 34 \\
& Idiopathic & 6 \\
& latrogenic & 22 \\
& Trauma & 4 \\
& Other & 2 \\
\hline
\end{tabular}

Table 2. Free functional muscle transfer selection

\begin{tabular}{|lc|}
\hline Flap type & No. of hemifaces \\
\hline Pectoralis minor & 64 \\
Latissimus dorsi & 24 \\
Gracilis & 8 \\
Pectoralis minor and platysma & 1 \\
Procedure abandoned & 1 \\
\hline
\end{tabular}

with $6 \%$ of patients in both groups lacking this recipient vessel.

Amongst the patients with a history of previous facial surgery or trauma, 33\% lacked a suitable facial artery and 44\% lacked a suitable facial vein, which was significantly more than amongst those who had no previous history of facial surgery or trauma $(\mathrm{P}<0.005)$.

Isolated analysis of the patients with acquired facial palsy with no history of trauma or surgery to the hemiface $(n=20)$ showed that only $6 \%$ lacked a facial artery and none had an absent facial vein.

\section{DISCUSSION}

Our series has shown that in $90 \%$ of all cases the facial artery is available as the recipient vessel and that $83 \%$ of patients have a facial vein suitable for use. A history of congenital facial palsy and previous facial trauma or surgery to the ipsilateral hemiface were factors that predicted these vessels were significantly more likely to be absent.

In circumstances when the facial artery and vein are not available, there are alternative vessels that can be utilised (Fig. 3). In one patient from our series of 87 it was necessary to abandon the procedure due to an absence of any suitable recipient vessels in the region.

The facial artery and vein act as our first choice recipient ves-

Table 3. Recipient artery used for each hemiface that was reanimated with free functional muscle transfer

\begin{tabular}{|lc|}
\hline Recipient artery & Number \\
\hline Facial & 88 \\
Superficial temporal & 3 \\
Lingual & 3 \\
Submandibular branch of facial artery & 1 \\
Superior thyroid & 1 \\
Superior laryngeal & 1 \\
Transverse facial & 0 \\
Procedure abandoned & 1 \\
\hline
\end{tabular}

Table 4. Recipient vein used for each hemiface that was reanimated with free functional muscle transfer

\begin{tabular}{|lc|}
\hline Recipient vein & Number \\
\hline Facial & 81 \\
Transverse facial & 9 \\
Submandibular branch of facial vein & 2 \\
Retromandibular & 2 \\
Superior thyroid & 1 \\
Superior laryngeal & 1 \\
Lingual & 1 \\
Procedure abandoned & 1 \\
Superficial temporal & 0 \\
\hline
\end{tabular}




\section{Fig. 3. Vessel selection algorithm}

An algorithm for recipient vessel selection in cases of free functional muscle transfer for facial reanimation. Other recipient vessels include the lingual, submandibular, superior thyroid and superior laryngeal artery/vein.

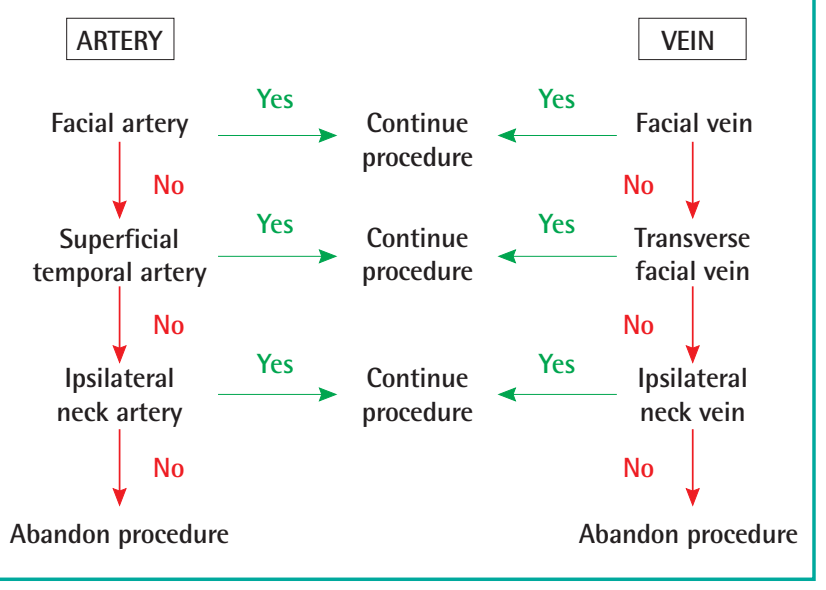

sels in cases of facial reanimation with free functional muscle transfer due to their locality to the muscle pedicle after inset, their calibre and good handling characteristics. After arising from the external carotid artery, the facial artery runs around the lower border of the mandible just anterior to the masseter muscle and then ascends towards the corner of the mouth in a subcutaneous plane. As such, it is readily identified during dissection towards the modiolus of the mouth. In a recent series of 201 cadaveric dissections, the facial artery was only absent in $2 \%$ of cases [6]. Earlier studies have shown that $100 \%$ of cadaveric dissections had a facial artery $[7,8]$. Our rate of $10 \%$ of patients lacking a facial artery is likely a result of our patient cohort including those with congenital facial palsy and a history of previous facial surgery/trauma. When analysing patients with acquired facial palsy and no history of facial surgery/trauma $(n=20)$, only one patient lacked a facial artery.

Although it was shown in these series $[6,8-10]$ that the branching pattern of the facial artery can vary widely, the initial path of travel from the anterior border of the masseter to the angle of the mouth was consistent. It is within this section of the facial artery that a suitable portion of vessel is selected for anastomosis in cases of free functional muscle transfer for facial reanimation.

The facial vein has been less widely studied. This vessel typically arises from the inner canthus of the eye from the confluence of the supraorbital and supratrochlear veins. The facial vein then descends down towards the angle of the jaw where it joins with the anterior branch of the retromandibular vein [9]. In the cadaveric series by Lohn et al. [5] the facial vein was absent in only $1 \%$ of cases, had a predictable course and was shown to be, on average, $0.7 \mathrm{~cm}$ more posterior from the gnathion than the facial artery. The proximity of the two vessels supports their use as first choice recipient artery and vein in cases of free tissue transfer for facial reanimation. Our finding of $18 \%$ of patients having an absent facial vein on one or both sides of the face is substantially higher than the findings from cadaveric studies, but can be explained by the cohort of patients included in our study. After exclusion of those with congenital facial palsy or a history of previous surgery/trauma to the hemiface none of the remaining patients lacked a facial vein.

In circumstances when the facial artery and/or vein are not present alternative recipient vessels should be selected (Fig. 1). In our unit, the majority of patients undergoing facial reanimation have a congenital aetiology and our first choice approach to these patients is to perform a two-stage procedure beginning with a cross-facial nerve graft coapted to the contralateral, healthy facial nerve. Our muscle of choice for the second stage is the pectoralis minor due to its fan-shape and minimal donor site morbidity [4]. Due to its short, centrally located vascular pedicle [6] donor vessels that are in close proximity facilitate direct microvascular anastomoses without the need for extensive donor vessel dissection or interpositional vascular grafts. As such, our second choice donor vein is the transverse facial vein. Cadaveric and living anatomical studies have shown that the transverse facial vein is dominant in cases of an absent facial vein $[5,10]$. This is consistent with findings in our study where $53 \%$ of patients that did not have a suitable facial vein had a transverse facial vein of sufficient calibre for use as the recipient vein. In the living anatomy series by Renshaw et al. [10] using colour Doppler ultrasound assessment, the transverse facial vein could not be located in $42 \%$ of participants. Although this study is limited by not being a cadaveric dissection study, these findings are important to consider when attempting to dissect out this vessels and support the rationale for not selecting this structure as the first choice recipient vein.

Although the transverse facial artery would be anatomically well-placed to be the second choice recipient artery, we have not had a case where this vessel is of suitable calibre to support the flap and subsequently it has not been included in the arterial arm of our algorithm. The transverse facial artery arises from the superficial temporal artery just before it exits the parotid gland after which it can branch in a multitude of different configurations [11]. It has previously been shown that in cases of facial artery hypoplasia the transverse facial artery can be of a larger calibre $[10,12]$. This, however, contradicts our findings where we found that when the facial artery was absent the transverse facial artery was also not available or suitable for use.

Given the unsuitability of the transverse facial artery, our second choice recipient artery is the superficial temporal artery. 
Both artery and vein run within the substance of the parotid gland and branch at, or above the level of the zygomatic arch [13]. To reach the position of the pedicle after inset of a pectoralis minor muscle flap the vessels must be dissected out over a sufficient length above and around the parotid gland to be turned down to reach the position of the muscle pedicle. In our series, these vessels were only used in combination with two latissimus dorsi and one gracilis free functional muscle flaps both of which provide longer pedicle length and, in the case of the gracilis muscle, have their pedicle orientated towards to the superficial temporal vessels. We note that other centres commonly use both the superficial temporal artery and vein as their recipient vessels of choice in facial reanimation procedures [14-16]. It should, however, be noted that in these studies the gracilis and latissimus dorsi flaps were the only muscles used for the reanimation, both of which provide a longer pedicle to reach the superficial temporal vessels and avoid the need to perform extensive dissection around the parotid gland. This would not be the case when using the pectoralis minor muscle for reanimation.

Although used in head and neck microsurgical reconstruction [17-20], it is our experience that the superficial temporal vein is thin-walled and friable during dissection, which is consistent with the experience of others [19]. The position of the vessels combined with the friable nature of the superficial temporal vein, risk of salivary fistula and short vascular pedicle on the pectoralis minor flap account for the superficial temporal vein not being included in our algorithm.

A final consideration, given the large proportion of our patient cohort with congenital facial palsy, is the importance of the superficial temporal vessels in ear reconstruction. This is because of the possible need for the pedicled temporoparietal fascial flap to be used when the reconstructed ear is elevated [21].

As shown in our study, it is very uncommon for there to be no suitable vessels from the facial, transverse facial, superficial temporal or ipsilateral neck territories. It is our recommendation to abandon the procedure if this is the case. To continue the operation would require the use of a contralateral neck vessel and interpositional vein grafts. The flap failure rate when using interpositional vein grafts in the head and neck has been reported to be between $25 \%$ to $35 \%$ [22-24]. Although there are functional and psychological benefits to successful facial reanimation, we feel that committing a patient the morbidity associated with exploration of the contralateral neck and vein graft harvest with a high risk of flap loss is not appropriate in facial reanimation cases where the principle gain is aesthetic.

It is not our current practice to perform routine preoperative vascular studies on patients undergoing facial reanimation. The first three vascular territories suggested in the algorithm can all be accessed via the rhytidectomy-type incision used to create the pocket where the muscle flap is inset [4]. If felt necessary, colour Doppler ultrasound could be considered in the preoperative work-up of these patients to aid operative planning and potentially reduce operative time $[10,25]$. Preoperative investigations would be of particular benefit in patients with congenital facial palsy or a history of previous facial surgery/trauma.

In cases when the facial artery and vein are absent, alternative recipient vessels are available to permit free functional muscle transfer for facial reanimation. Our algorithm can help to guide vessel selection in such cases and is most suited to when a pectoralis minor muscle is used to reanimate the face. Amongst patients with congenital facial palsy or in those with a previous history of facial surgery or trauma, the facial vessels are more likely to be absent and so the surgeon should then look towards the transverse facial or superficial temporal artery/vein as recipient structures.

\section{REFERENCES}

1. Ghali S, MacQuillan A, Grobbelaar AO. Reanimation of the middle and lower face in facial paralysis: review of the literature and personal approach. J Plast Reconstr Aesthet Surg 2011;64:423-31.

2. Thompson N. Autogenous free grafts of skeletal muscle. A preliminary experimental and clinical study. Plast Reconstr Surg 1971;48:11-27.

3. Harii K, Ohmori K, Torii S. Free gracilis muscle transplantation, with microneurovascular anastomoses for the treatment of facial paralysis. A preliminary report. Plast Reconstr Surg 1976;57:133-43.

4. Harrison DH, Grobbelaar AO. Pectoralis minor muscle transfer for unilateral facial palsy reanimation: an experience of 35 years and 637 cases. J Plast Reconstr Aesthet Surg 2012;65:845-50.

5. Lohn JW, Penn JW, Norton J, et al. The course and variation of the facial artery and vein: implications for facial transplantation and facial surgery. Ann Plast Surg 2011;67:184-8.

6. MacQuillan A, Horlock N, Grobbelaar A, et al. Arterial and venous anatomical features of the pectoralis minor muscle flap pedicle. Plast Reconstr Surg 2004;113:872-6.

7. Niranjan NS. An anatomical study of the facial artery. Ann Plast Surg 1988;21:14-22.

8. Koh KS, Kim HJ, Oh CS, et al. Branching patterns and symmetry of the course of the facial artery in Koreans. Int $\mathrm{J}$ Oral Maxillofac Surg 2003;32:414-8.

9. Houseman ND, Taylor GI, Pan WR. The angiosomes of the head and neck: anatomic study and clinical applications. 
Plast Reconstr Surg 2000;105:2287-313.

10. Renshaw A, Whitwell KA, Berger L, et al. The use of color Doppler ultrasound in the assessment of vessels for facial transplantation. Ann Plast Surg 2007;59:82-6.

11. Yang HJ, Gil YC, Lee HY. Topographical anatomy of the transverse facial artery. Clin Anat 2010;23:168-78.

12. Soikkonen K, Wolf J, Hietanen J, et al. Three main arteries of the face and their tortuosity. Br J Oral Maxillofac Surg 1991;29:395-8.

13. Chen TH, Chen CH, Shyu JF, et al. Distribution of the superficial temporal artery in the Chinese adult. Plast Reconstr Surg 1999;104:1276-9.

14. Bianchi B, Copelli C, Ferrari S, et al. Facial animation with free-muscle transfer innervated by the masseter motor nerve in unilateral facial paralysis. J Oral Maxillofac Surg 2010;68: 1524-9.

15. Takushima A, Harii K, Asato H, et al. Neurovascular freemuscle transfer for the treatment of established facial paralysis following ablative surgery in the parotid region. Plast Reconstr Surg 2004;113:1563-72.

16. Takushima A, Harii $\mathrm{K}$, Asato $\mathrm{H}$, et al. Neurovascular freemuscle transfer to treat facial paralysis associated with hemifacial microsomia. Plast Reconstr Surg 2002;109:1219-27.

17. Nahabedian MY, Singh N, Deune EG, et al. Recipient vessel analysis for microvascular reconstruction of the head and neck. Ann Plast Surg 2004;52:148-55.
18. Takamatsu A, Harashina T, Inoue T. Selection of appropriate recipient vessels in difficult, microsurgical head and neck reconstruction. J Reconstr Microsurg 1996;12:499-507.

19. Shimizu F, Lin MP, Ellabban M, et al. Superficial temporal vessels as a reserve recipient site for microvascular head and neck reconstruction in vessel-depleted neck. Ann Plast Surg 2009;62:134-8.

20. Chia HL, Wong CH, Tan BK, et al. An algorithm for recipient vessel selection in microsurgical head and neck reconstruction. J Reconstr Microsurg 2011;27:47-56.

21. Nagata S. A new method of total reconstruction of the auricle for microtia. Plast Reconstr Surg 1993;92:187-201.

22. Schusterman MA, Miller MJ, Reece GP, et al. A single center's experience with 308 free flaps for repair of head and neck cancer defects. Plast Reconstr Surg 1994;93:472-8.

23. Jones NF, Johnson JT, Shestak KC, et al. Microsurgical reconstruction of the head and neck: interdisciplinary collaboration between head and neck surgeons and plastic surgeons in 305 cases. Ann Plast Surg 1996;36:37-43.

24. Podrecca S, Salvatori P, Squadrelli Saraceno M, et al. Review of 346 patients with free-flap reconstruction following head and neck surgery for neoplasm. J Plast Reconstr Aesthet Surg 2006;59:122-9.

25. Zhao Z, Li S, Xu J, et al. Color Doppler flow imaging of the facial artery and vein. Plast Reconstr Surg 2000;106:124953. 